\section{BRAZIULIAN JOURNAL \\ OF MEDICAL AND BIOLOGICAL RLSF.ARCH}

www.bjournal.com.br
ISSN 0100-879X

Volume 42 (12) 1119-1247 December 2009

BIOMEDICAL SCIENCES

AND

CLINICAL INVESTIGATION

Braz J Med Biol Res, December 2009, Volume 42(12) 1242-1247

Pattern of functional antibody activity against Haemophilus influenzae type $b$ (Hib) in infants immunized with diphtheriatetanus-pertussis/Hib Brazilian combination vaccine

D.C.S. Matos, A.M.V. Silva, P.C.C. Neves, R.M. Martins, A. Homma and R. Marcovistz

The Brazilian Journal of Medical and Biological Research is partially financed by
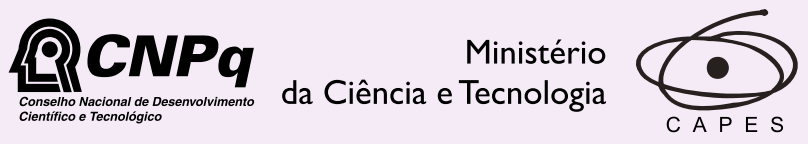

Ministério da Educação

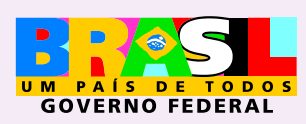

Institutional Sponsors 


\title{
Pattern of functional antibody activity against Haemophilus influenzae type b (Hib) in infants immunized with diphtheria-tetanus-pertussis/Hib Brazilian combination vaccine
}

\author{
D.C.S. Matos ${ }^{1}$, A.M.V. Silva ${ }^{1}$, P.C.C. Neves ${ }^{1}$, R.M. Martins ${ }^{2}$, \\ A. Homma ${ }^{3}$ and R. Marcovistz ${ }^{1}$ \\ ${ }^{1}$ Laboratório de Tecnologia Imunológica, ${ }^{2}$ Assessoria Clínica, Bio-Manguinhos, \\ ${ }^{3}$ Diretoria de Bio-Manguinhos, Fiocruz, Rio de Janeiro, RJ, Brasil
}

\begin{abstract}
We evaluated the functional activity of Haemophilus influenzae B (Hib) antibodies elicited in a group of infants immunized with the diphtheria-tetanus-pertussis vaccine combined with an Hib vaccine produced totally in Brazil after technological transfer of Hib vaccine production from Glaxo SmithKline, Belgium. Blood samples from immunized infants $(N=985)$ were collected for the determination of Hib antibodies. Total Ig and IgM and IgG subclasses of antibodies against polyribosyl ribitol phosphate (PRP) were analyzed by ELISA. Almost all vaccinees $(97.56 \%, 961 / 985)$ developed a strong anti-PRP IgG antibody response $(\geq 1.0$ $\mu \mathrm{g} / \mathrm{mL})$, while an anti-PRP IgM response was observed in $64.24 \%(634 / 985)$ of them $(\geq 0.15 \mu \mathrm{g} / \mathrm{mL})$. Only $18.88 \%(186 / 985)$ of the infants in the group with high PRP antibody $\lg G$ concentrations $(\geq 1.0 \mu \mathrm{g} / \mathrm{mL})$ developed a high IgM antibody response. Anti-PRP IgG antibody levels were significantly higher than anti-PRP IgM. These results demonstrate the predominance of IgG antibodies over IgM antibodies in response to PRP, with a ratio of 17:1. IgG antibodies were predominantly of the IgG1 subclass. An increase in IgG avidity was also observed during the course of immunization.
\end{abstract}

Key words: DTP-Hib vaccine; Haemophilus influenzae type b; Antibody avidity; PRP immune response

\section{Introduction}

Since 1998 the WHO has recommended that Haemophilus influenzae type $\mathrm{b}(\mathrm{Hib})$ vaccination be included in routine infant immunization programs, according to country capacities, preferably as a diphtheria-tetanus-pertussis (DTP)-based combination (1). However, the immunological response, which insures the improved immunogenicity and priming induced by conjugated vaccines, is still only partially understood. It is known that $\lg G$ and IgM antibodies against the capsular polysaccharide polyribosyl ribitol phosphate (PRP) are very important for the protective immunity to Hib, and that the covalent linkage of PRP to any protein is also important for the enhanced immunogenicity of glycoconjugated vaccines (2-4).

The response to conjugated vaccine is known to be thymus dependent ( $T$ cell dependent), thus permitting memory responses even in infants. In contrast, molecules such as
PRP, a thymus-independent class 2 antigen (TI-2 antigen), can activate only mature $B$ cells, preventing infants from producing antibodies against polysaccharide epitopes in an efficient manner. The immune systems of young children are immature and do not respond effectively to carbohydrate antigens before about 5 years of age. On the other hand, most physiological TI-2 responses occur in the spleen, the marginal zone $B$ cells, which are rare at birth and which accumulate with age (5-11). Thus, the development of vaccines with PRP conjugated with a protein carrier was very important and has reduced the recommended minimum age of 5 years to 6 months for this vaccination (5).

Over the years, new technologies for combined vaccines have been developed and several different $\mathrm{Hib}$ vaccines have been licensed after extensive safety and immunogenicity evaluation. At least four different PRP-conjugated vac-

Correspondence: R. Marcovistz, Bio-Manguinhos, Fiocruz, Av. Brasil, 4365, Pavilhão Rocha Lima, 21040-360 Rio de Janeiro, RJ, Brasil. Fax: +55-21-2260-4727. E-mail: rugimar@bio.fiocruz.br

Research supported by FINEP, Ministério da Ciência e Tecnologia, Bio-Manguinhos-Fiocruz.

Received May 20, 2009. Accepted August 19, 2009. Available online November 6, 2009. Published December 4, 2009. 
cines are available, according to the carrier protein: conjugation with diphtheria toxoid (PRP-D), or an avirulent mutant of the diphtheric toxin (PRP-CRM), PRP conjugated with tetanus toxoid (PRP-T), and PRP conjugated with the outer membrane protein of Neisseria meningitidis (PRP-OMP) (12-14).

Conjugated vaccines have not only been able to induce an immune response in young infants, but have also been able to induce a memory immune response and to promote a secondary boost response with either conjugated or unconjugated PRP vaccines. Vaccine trials have shown the ability of the conjugated vaccines to activate and prime the previously immature, non-responsive immunity of infants $(15,16)$.

In 2006, a randomized, double-blind study was designed to evaluate the immunogenicity, safety and consistency of production of a combined DTP-Hib vaccine totally produced in Brazil (DTP/Hib-BM) after a technological transfer of Hib production from Glaxo SmithKline (GSK), Belgium.

Overall, 1000 infants were recruited and received vaccine doses at 2, 4, and 6 months of age. Regarding the Hib component, there was $100 \%$ seroprotection $(\geq 0.15 \mu \mathrm{g} / \mathrm{mL})$, and there was no clinically relevant difference in reactogenicity between the 3 lots of Brazilian DTP/Hib studied and the reference vaccine lot with Hib produced by GSK (17).

The objective of the present study was to analyze the classes and subclasses as well as the functional activity of antibodies to Hib elicited in infants immunized with DTP/HibBM or DTP/Hib-GSK vaccines.

\section{Subjects and Methods}

\section{Subjects and vaccines}

This study is a follow-up of a previously reported clinical trial in which the immunogenicity, reactivity and consistency of production of DTP/Hib-BM was assessed (17). One thousand infants were immunized according to the immunization program of the Brazilian Health Ministry in a double-blind and randomized study approved by the National Research Ethics Committee, the Ethics Committee of Fundação Oswaldo Cruz and of the Municipal Health Secretary of Rio de Janeiro, and by the National Regulatory Agency. The protocol was registered under Standard Randomized Clinical Trial Number 67676353 (17).

\section{Serum samples}

Blood samples from 985 infants were collected for evaluation of lg, IgG, and IgM PRP antibodies. In a subset of 300 infants, randomly chosen sera collected before and 1 month after the 3rd vaccine dose were used in comparative studies. Serum samples from the same infant were tested in parallel.

\section{Antibody assay}

$\lg$, IgM, and IgG subclasses of antibodies against PRP were analyzed by ELISA (3-6). Briefly, each well of a microplate (Maxisorb; Nunc, USA) was coated with $0.4 \mu \mathrm{g} /$
$\mathrm{mL}$ PRP-tyramine antigen in $0.1 \mathrm{~mL} 0.05 \mathrm{M}$ carbonate buffer, $\mathrm{pH} 9.6$, overnight at $4^{\circ} \mathrm{C}$. Plates were washed with PBS plus $0.05 \%$ Tween 20 (PBS-Tween), and blocked with 0.1 $\mathrm{mL}$ PBS containing $0.5 \%$ bovine serum albumin (BSA) for $1 \mathrm{~h}$ at $37^{\circ} \mathrm{C}$. Sera were serially diluted 2 -fold in $0.5 \%$ PBSTween-BSA starting at 1:10 dilution and up to 1:1280 dilution and the plates were incubated for $1 \mathrm{~h}$ at $37^{\circ} \mathrm{C}$. After washing three times with PBS-Tween, $0.1 \mathrm{~mL} /$ well of a 1/500 dilution of mouse anti-human IgG conjugated with horseradish peroxidase (HRP, BD-Pharmingen, USA) or a 1/30,000 dilution of biotinylated mouse anti-human IgM (BD-Pharmingen) in PBS-Tween-BSA was added and the plates were incubated again for $1 \mathrm{~h}$ at $37^{\circ} \mathrm{C}$. For IgM antibody detection, a streptavidin HRP-conjugate (BD-Pharmingen) was added to the plates for $1 \mathrm{~h}$ at $37^{\circ} \mathrm{C}$. IgG1 and IgG2 anti-PRP antibody titers were determined using specific monoclonal antibodies (BD-Pharmingen). Next, the tetramethylbenzidine substrate (Sigma, USA) was added and the reaction stopped with 2 $\mathrm{M} \mathrm{NaOH} 15$ min later. Absorbance was measured at 450 $\mathrm{nm}$ with an ELISA microreader (Sunrise; Tecan, Germany). Standard curves were generated using human $\lg$, IgG1, and IgG2 and IgM immunoglobulins. The ELISA antibody titer curves for each serum specimen were analyzed by a four-parameter logistic curve-fitting technique (Softmax Pro version 5, Molecular Devices, USA). The minimum level of detection of this assay was estimated to be $0.10 \mu \mathrm{g} / \mathrm{mL}$ lg at a 1:10 dilution of the tested serum.

\section{IgG antibody avidity assay}

Anti-PRP IgG avidity was measured by ELISA by the method of Agbarakwe et al. (16) with few modifications (6). Briefly, plates were coated with PRP and saturated as explained above, duplicate serum samples were then diluted to 1:20 in PBS-Tween-BSA and plates were incubated at $37^{\circ} \mathrm{C}$ for $1 \mathrm{~h}$ followed by three washes with PBS-Tween. Next, 100 $\mu \mathrm{L}$ 3.0 $\mathrm{M}$ urea was added to only one of the serum dilution rows, while $100 \mu \mathrm{LPBS}-T$ ween was added to the other serum dilutions, corresponding to the control without urea. After 15 min of incubation, plates were washed with PBS-Tween for removal of both the unbound antibodies and the chaotropic agent by washing. The remaining bound IgG antibodies were detected with peroxidase-conjugated anti-human IgG in a manner similar to that described above.

The avidity index (Al) was calculated as the mean absorbance of reactions in which the antibodies were exposed to urea divided by the mean absorbance of reactions in which the antibodies were not exposed to urea, and expressed as percentage. The avidity assay could not be evaluated for sera with $<0.5 \mu \mathrm{g} / \mathrm{mL}$ IgG anti-PRP antibody. Samples with an Al below $49 \%$ were considered to have low avidity and samples with an Al of 50 to $79 \%$ and of more than $80 \%$ were considered to have medium and high avidity, respectively.

\section{Statistical analyses}

The paired nonparametric Wilcoxon test was used to 
compare anti-PRP antibody titers and AI between vaccination time points (before and 1 month after the 3 rd dose). AP value of $<0.05$ was considered to be statistically significant. Spearman's correlation coefficient was used to evaluate the relationship between $\lg G$ subclasses and lgG avidity.

\section{Results}

\section{Anti-PRP classes and subclass antibodies}

Anti-PRP Ig total, IgG and IgM antibody titers were evaluated in serum samples obtained from children 1 month after the 3rd dose of DTP-Hib vaccine and are summarized in Table 1. All infants were seroprotected against PRP-T after the complete vaccination schedule, and $97.25 \%$ (958/985) of them presented high concentrations of total anti-PRP Ig $(\geq 1.0 \mu \mathrm{g} / \mathrm{mL})$. Almost all vaccinees $(97.56 \%$, 961/985) developed a strong IgG anti-PRP antibody response $(\geq 1.0 \mu \mathrm{g} / \mathrm{mL})$, while an anti-PRP IgM response was observed in $64.24 \%(634 / 985)$ of them $(\geq 0.15 \mu \mathrm{g} / \mathrm{mL})$. Only $18.88 \%$ (186/985) of the infants developed high levels of IgM antibody in the group with high PRP antibody IgG concentrations $(\geq 1.0 \mu \mathrm{g} / \mathrm{mL})$. The levels of anti-PRP IgG antibodies were significantly higher than the levels of antiPRP IgM antibodies ( $P<0.0001)$, with IgG levels ranging from 0.17 to $163 \mu \mathrm{g} / \mathrm{mL}$ (mean $=16.91 \pm 0.62$ ) and $\operatorname{lgM}$ levels ranging from 0.10 to $18 \mu \mathrm{g} / \mathrm{mL}$ (mean $=0.88 \pm 0.05$; Figure $1 \mathrm{~A}$ ). These results demonstrate the predominance of IgG antibodies over IgM antibodies in response to PRP, with a ratio of $17: 1$.

Figure 1B shows the geometric means (GM) of specific IgG levels before and one month after the 3rd vaccine dose in all volunteers. As expected, a significant increase $(P<$ $0.0001)$ of PRP IgG levels was observed one month after the 3rd vaccine dose $(\mathrm{GM}=10.16 \mu \mathrm{g} / \mathrm{mL}, 95 \% \mathrm{Cl}=15.6-18.12$ $\mu \mathrm{g} / \mathrm{mL}$ ) compared to PRP IgG levels before the last dose of vaccine $(\mathrm{GM}=5.03 \mu \mathrm{g} / \mathrm{mL}, 95 \% \mathrm{Cl}=5.87-6.63 \mu \mathrm{g} / \mathrm{mL})$.

The DTP-Hib vaccine induced a definitely predominant IgG1 response since $90 \%$ of IgG antibodies belonged to the IgG1 subclass. Anti-PRP IgG1 levels were significantly higher than anti-PRP IgG2 levels $(11.13 \pm 0.51$ vs $4.23 \pm$ $0.24 \mu \mathrm{g} / \mathrm{mL}, \mathrm{P}<0.0001$ ) as shown in Figure $1 \mathrm{C}$.

\section{IgG antibody avidity}

There was an increase in IgG antibody avidity during the course of immunization. In fact, the IgG Al were significantly higher in sera obtained from infants one month after the complete schedule of vaccination than in sera obtained before the 3 rd vaccine dose $(P<0.0001)$, as shown in Figure $2 \mathrm{~A}$. The avidity percentage of absorbance values in sera, following 2 doses of DTP-Hib vaccine was $70 \%(95 \% \mathrm{Cl}=68-73 \%)$. After the 3rd dose, the mean IgG $\mathrm{Al}$ in the group was $80 \%(95 \% \mathrm{Cl}=78-83 \%)$, indicating a significant increase $(P<0.0001)$.

The pattern antibody avidity distribution induced against Hib during the immunization process presented different

Table 1. Anti-PRP antibodies in infants one month after the third DTP/Hib vaccine dose.

\begin{tabular}{lccc}
\hline $\begin{array}{l}\text { Anti-PRP antibody concentration } \\
(\mu \mathrm{g} / \mathrm{mL})\end{array}$ & $\begin{array}{c}\mathrm{Ig} \\
(\%)\end{array}$ & \multicolumn{1}{l|}{$\begin{array}{l}\mathrm{lgG} \\
(\%)\end{array}$} & \multicolumn{1}{c}{$\begin{array}{l}\mathrm{IgM} \\
(\%)\end{array}$} \\
\hline$\leq 0.14$ & 0.00 & 0.20 & 16.75 \\
$0.15-0.99$ & 2.75 & 2.24 & 64.37 \\
$\geq 1.00$ & 97.25 & 97.56 & 18.88 \\
\hline
\end{tabular}

$\mathrm{PRP}=$ polyribosyl ribitol phosphate; $\mathrm{DTP} / \mathrm{Hib}=$ diphtheria-tetanus-pertussis/Haemophilus influenzae B.
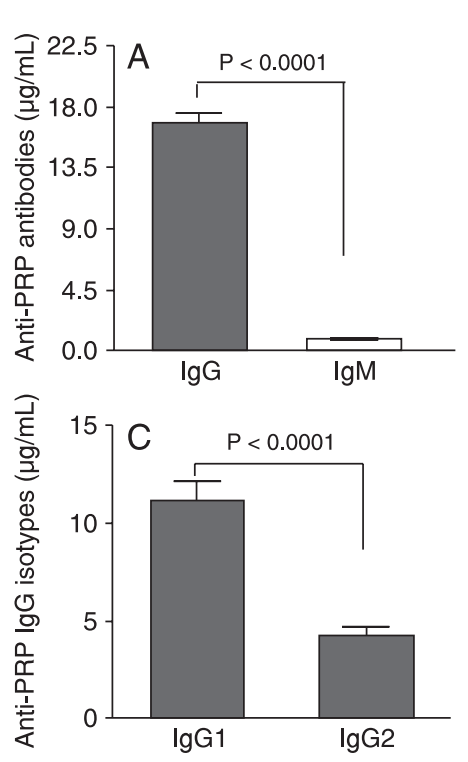

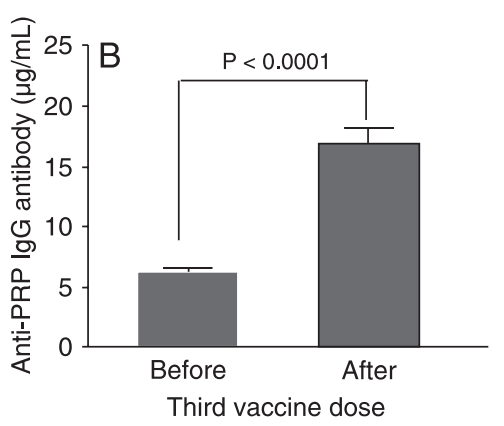

Third vaccine dose
Figure 1. Concentration of anti-PRP antibodies in sera of immunized children. The bars indicate the geometric mean of antibody levels and 95\% confidence intervals. Statistical comparisons were made with the Wilcoxon test. A, Comparison of anti-PRP $\lg$ and $\operatorname{lgM}$ concentrations in the sera one month after the 3rd DTP/Hib vaccine dose. B, Comparison of anti-PRP IgG concentration before and one month after the 3 rd DTP/Hib vaccine dose. C, IgG subclasses one month after the 3rd DTP/Hib vaccine dose. $\mathrm{PRP}=$ polyribosyl ribitol phosphate; DTP/Hib = Diphtheria-tetanus-pertussis/Haemophilus influenzae B. 

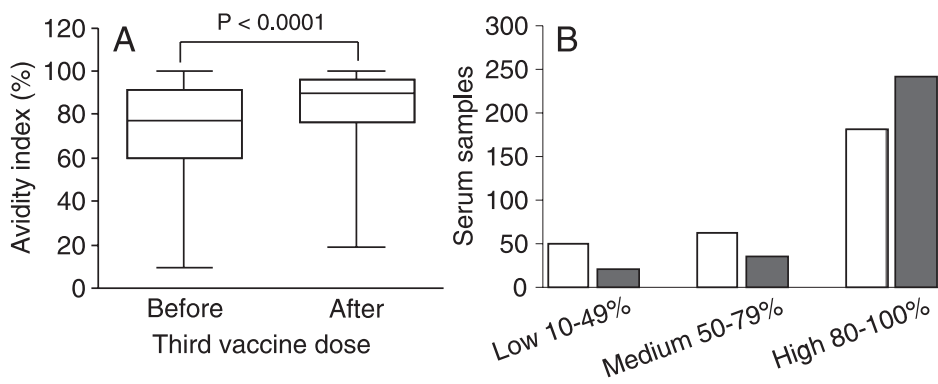

Figure 2. Avidity index and distribution of PRP $\lg G$ antibodies in serum samples from children receiving DTP-Hib vaccination. A. Avidity index before and 1 month after the 3rd DTP-Hib vaccine dose in 300 infants. Statistical comparison was made with the Wilcoxon test. $B$. Distribution of the avidity of anti-PRP IgG antibodies in a group of 300 infants immunized with DTP/Hib. Open and filled bars represent blood sampling before and 1 month after the 3rd dose of the DTP/Hib vaccine, respectively. PRP = polyribosyl ribitol phosphate; DTP/Hib = diphtheria-tetanus-pertussis/Haemophilus influenzae B.

profiles of avidity intensity before and after the 3rd dose (Figure 2B).

\section{Discussion}

In this study, we quantitatively and qualitatively evaluated the specific anti-Hib response induced by the Hib PRP-T conjugated vaccine during a clinical trial of the DTP-Hib vaccine produced by Bio-Manguinhos/Fiocruz, Brazil, after technological transfer from Glaxo-SmithKline.

High-affinity antibodies are required to neutralize the infectivity of most pathogens. Therefore, effective vaccines against these microorganisms must induce affinity maturation and memory $\mathrm{B}$ cell formation, and both these events will occur only if the vaccines are able to activate $T$ helper cells. This concept has been applied to the design of vaccines against some bacterial infections in which the target antigen is a capsular polysaccharide, which is incapable of stimulating T cells. This is also the case of the polysaccharide from Hib which, when covalently linked to tetanus toxoid, forms the equivalent of an hapten-carrier conjugate, which does activate $\mathrm{T}$ helper cells. Thus, the antibody response induced by this conjugate vaccine requires the participation of $\mathrm{T}$ cells that may consequently stimulate the subclass switching, affinity maturation and memory with long-lived protective immunity in B lymphocytes. In contrast, the antibodies produced in the absence of $\mathrm{T}$ helper cells by polysaccharide vaccines not conjugated to proteins are generally of low affinity and consist mainly of $\operatorname{lgM}$ and $\lg G 2$ (18).

The humoral immune response to Hib conjugated vaccines is usually evaluated by measuring serum anti-PRP antibodies and by the Al. Avidity of anti-PRP IgG has been tested by ELISA-based elution assays $(4,19-24)$, which have demonstrated that PRP conjugates elicit high-avidity antibodies, with increased functional activity $(4,18)$.
We observed here that $97.25 \%$ of the infants immunized with DTP-Hib produced high levels $(\geq 1.0 \mu \mathrm{g} / \mathrm{mL}$ ) of PRP IgG antibodies, considered to be a serological surrogate of long-term protection. Some researchers have already established that protection from Hib disease is correlated with the presence of antibodies to PRP, and have considered $0.15 \mu \mathrm{g} / \mathrm{mL}$ of anti-PRP to be the minimum level of antibodies for short-term protection against Hib disease and $1 \mu \mathrm{g} / \mathrm{mL}$ to be the minimum level for long-term protection $(25,26)$. Regarding the IgM antibody, our results showed that almost all vaccinees produced anti-PRP IgM, but at low levels compared to anti-IgG antibodies. The production of IgM antibodies against PRP induced by DTP-Hib, although small, plays an important role in opsonization and phagocytosis by activation of the classical complement pathway, an important defense mechanism against bacteria with polysaccharide-rich capsules, such as Haemophilus. The IgM antibody, due to its pentameric structure, is a more efficient complement-binding antibody than IgG.

When we assessed the subclasses of IgG we observed that the vaccinees responded with IgG anti-PRP of both IgG1 and IgG2 subclasses, but with a significant predominance of anti-PRP IgG1. We observed a good relationship between IgG and IgG1 antibody levels, but no correlation was found with IgG2 levels. Our results agree with those obtained with different PRP conjugates by Granoff et al. (27), who also reported that infants immunized with PRP-OMP respond predominantly with anti-PRP $\lg G 1$, while adults immunized with unconjugated PRP or PRP-D respond with anti-PRP of both IgG1 and IgG2 subclasses, as is the case for the DTP-Hib vaccine. Hetherington et al. (28) have also suggested that the IgG subclass response to PRP protein conjugate is age independent. Other investigators have also reported that IgG1 antibodies are more effective than IgG2 antibodies for Hib in an opsonization test and in activating complement-mediated bacteriolysis (29-32).

The present results demonstrate that antibody avidity was increased after the third immunization with the DTP-Hib vaccine. The increased Al between the 4th and 7th month of age indicates that memory responses must have occurred during DTP-Hib vaccination in Brazilian infants. These results might be due to the vaccine reactivation of earlier induced memory B cells, as reported by Hougs et al. (33). Goldblatt et al. (18) have demonstrated that antibody avidity was lower in infants with anti-PRP antibody concentrations below $1.0 \mu \mathrm{g} / \mathrm{mL}$, indicating the absence of priming when antibody concentrations are low. Amir et al. (29) also evaluated the functional antibody activity of vaccines induced by $\mathrm{Hib}$ and found the avidity of antibodies to be an essential factor for serum bactericidal activities.

The increase in avidity reflects the process of somatic 
hypermutation of immunoglobulin genes and the subsequent selection resulting in high affinity $B$ cells that occurs in the germinal center following a T cell-dependent response (34). Thus, the Al has been used as a surrogate marker of immunological priming since it also relies on a $\mathrm{T}$ celldependent response (18).

The present study shows that the Brazilian DTP/Hib vaccine demonstrated strong immunogenicity after 2 doses, with most infants producing high levels of $\lg G$ antibodies, but the results were even better one month after the 3rd vaccine dose. When analyzing the antibody Al, we were able to demonstrate that high avidity PRP antibodies were found after the 2nd DTP/Hib vaccine dose, and avidity had improved after the third dose. Although two vaccine doses induce antibodies with a protection level above $1 \mu \mathrm{g} / \mathrm{mL}$, the 3rd dose significantly improves the functional activity of

\section{References}

1. Global Programme for Vaccines and Immunization (GPV). The WHO position paper on Haemophilus influenzae type $b$ conjugate vaccines. Wkly Epidemiol Rec 1998; 73: 64-68.

2. Kelly DF, Moxon ER, Pollard AJ. Haemophilus influenzae type b conjugate vaccines. Immunology 2004; 113: 163174.

3. Anderson P, Pichichero M, Insel R, Farsad P, Santosham M. Capsular antigens noncovalently or covalently associated with protein as vaccines to Haemophilus influenzae type b: comparison in two-year-old children. J Infect Dis 1985; 152: 634-636.

4. Romero-Steiner S, Holder PF, Gomez de Leon P, Spear W, Hennessy TW, Carlone GM. Avidity determinations for Haemophilus influenzae type $b$ anti-polyribosylribitol phosphate antibodies. Clin Diagn Lab Immunol 2005; 12: 1029-1035.

5. McCool TL, Harding CV, Greenspan NS, Schreiber JR. Band T-cell immune responses to pneumococcal conjugate vaccines: divergence between carrier- and polysaccharidespecific immunogenicity. Infect Immun 1999; 67: 48624869.

6. Phan TG, Gardam S, Basten A, Brink R. Altered migration, recruitment, and somatic hypermutation in the early response of marginal zone B cells to T cell-dependent antigen. $\mathrm{J}$ Immunol 2005; 174: 4567-4578.

7. Martin F, Kearney JF. Marginal-zone B cells. Nat Rev Immunol 2002; 2: 323-335.

8. Delemarre FG, Claassen E, Van Rooijen N. Primary in situ immune response in popliteal lymph nodes and spleen of mice after subcutaneous immunization with thymus-dependent or thymus-independent (type 1 and 2) antigens. Anat Rec 1989; 223: 152-157.

9. Lentz VM, Manser T. Cutting edge: germinal centers can be induced in the absence of T cells. J Immunol 2001; 167: 15-20.

10. Willenbrock K, Jungnickel B, Hansmann ML, Kuppers R. Human splenic marginal zone B cells lack expression of activation-induced cytidine deaminase. Eur J Immunol 2005; 35: 3002-3007.

11. Wu ZQ, Khan $A Q$, Shen $Y$, Schartman J, Peach R, Lees A, et antibodies against Haemophilus influenzae, thus ensuring effective long-term protection against Hib.

Finally, we emphasize that the combined DTP/Hib vaccine allowed a reduction in the number of required injections, thus improving compliance with the vaccination schedule and higher vaccination coverage. Moreover, the combined vaccine reduced the logistic costs related to factors such as number of visits to health care centers.

\section{Acknowledgments}

The authors would like to thank the mothers, guardians and all partners inside and outside of Bio-Manguinhos who participated in the clinical study of Brazilian DTP-Hib vaccine in 2007. al. B7 requirements for primary and secondary protein- and polysaccharide-specific Ig isotype responses to Streptococcus pneumoniae. J Immunol 2000; 165: 6840-6848.

12. Peltola $\mathrm{H}$. Worldwide Haemophilus influenzae type $\mathrm{b}$ disease at the beginning of the 21st century: global analysis of the disease burden 25 years after the use of the polysaccharide vaccine and a decade after the advent of conjugates. Clin Microbiol Rev 2000; 13: 302-317.

13. Mulholland K, Hilton S, Adegbola R, Usen S, Oparaugo A, Omosigho C, et al. Randomised trial of Haemophilus influenzae type-b tetanus protein conjugate vaccine [corrected] for prevention of pneumonia and meningitis in Gambian infants. Lancet 1997; 349: 1191-1197.

14. Lagos R, Horwitz I, Toro J, San Martin O, Abrego P, Bustamante $\mathrm{C}$, et al. Large scale, postlicensure, selective vaccination of Chilean infants with PRP-T conjugate vaccine: practicality and effectiveness in preventing invasive Haemophilus influenzae type b infections. Pediatr Infect Dis J 1996; 15: 216-222.

15. Claesson BA, Trollfors B, Lagergard T, Taranger J, Bryla D, Otterman G, et al. Clinical and immunologic responses to the capsular polysaccharide of Haemophilus influenzae type $b$ alone or conjugated to tetanus toxoid in 18- to 23-month-old children. J Pediatr 1988; 112: 695-702.

16. Agbarakwe AE, Griffiths $H$, Begg N, Chapel HM. Avidity of specific IgG antibodies elicited by immunisation against Haemophilus influenzae type b. J Clin Pathol 1995; 48: 206209.

17. Martins Rde M, Camacho LA, Marcovistz R, Noronha TG, Maia Mde L, dos Santos EM, et al. Immunogenicity, reactogenicity and consistency of production of a Brazilian combined vaccine against diphtheria, tetanus, pertussis and Haemophilus influenzae type b. Mem Inst Oswaldo Cruz 2008; 103: 711-718.

18. Goldblatt $D, V a z A R$, Miller E. Antibody avidity as a surrogate marker of successful priming by Haemophilus influenzae type $\mathrm{b}$ conjugate vaccines following infant immunization. $J$ Infect Dis 1998; 177: 1112-1115.

19. Pichichero ME, Voloshen T, Zajac D, Passador S. Avidity 
maturation of antibody to Haemophilus influenzae type b (Hib) after immunization with diphtheria-tetanus-acellular pertussis-hib-hepatitis B combined vaccine in infants. $J$ Infect Dis 1999; 180: 1390-1393.

20. Plikaytis BD, Holder PF, Pais LB, Maslanka SE, Gheesling LL, Carlone GM. Determination of parallelism and nonparallelism in bioassay dilution curves. J Clin Microbiol 1994; 32: 2441-2447.

21. Poolman J, Kaufhold A, De Grave D, Goldblatt D. Clinical relevance of lower Hib response in DTPa-based combination vaccines. Vaccine 2001; 19: 2280-2285.

22. Romero-Steiner S, Fernandez J, Biltoft C, Wohl ME, Sanchez J, Feris J, et al. Functional antibody activity elicited by fractional doses of Haemophilus influenzae type b conjugate vaccine (polyribosylribitol phosphate-tetanus toxoid conjugate). Clin Diagn Lab Immunol 2001; 8: 1115-1119.

23. Schlesinger Y, Granoff DM. Avidity and bactericidal activity of antibody elicited by different Haemophilus influenzae type b conjugate vaccines. The Vaccine Study Group. JAMA 1992; 267: 1489-1494.

24. Singleton R, Hammitt L, Hennessy T, Bulkow L, DeByle C, Parkinson A, et al. The Alaska Haemophilus influenzae type $b$ experience: lessons in controlling a vaccine-preventable disease. Pediatrics 2006; 118: e421-e429.

25. Kayhty H, Peltola H, Karanko V, Makela PH. The protective level of serum antibodies to the capsular polysaccharide of Haemophilus influenzae type b. J Infect Dis 1983; 147: 1100.

26. Santosham M, Reid R, Ambrosino DM, Wolff MC, AmeidoHill J, Priehs C, et al. Prevention of Haemophilus influenzae type $\mathrm{b}$ infections in high-risk infants treated with bacterial polysaccharide immune globulin. N Engl J Med 1987; 317: 923-929.

27. Granoff DM, Weinberg GA, Shackelford PG. IgG subclass response to immunization with Haemophilus influenzae type $b$ polysaccharide-outer membrane protein conjugate vaccine. Pediatr Res 1988; 24: 180-185.

28. Hetherington SV, Rutkowski AF. Antibody affinity in infants after immunization with conjugated capsular polysaccharide from Haemophilus influenzae type b. J Infect Dis 1990; 162: 1185-1188.

29. Amir J, Scott MG, Nahm MH, Granoff DM. Bactericidal and opsonic activity of IgG1 and IgG2 anticapsular antibodies to Haemophilus influenzae type b. J Infect Dis 1990; 162: 163-171.

30. Bredius RG, de Vries CE, Troelstra A, van Alphen L, Weening RS, van de Winkel JG, et al. Phagocytosis of Staphylococcus aureus and Haemophilus influenzae type B opsonized with polyclonal human $\lg \mathrm{G} 1$ and $\lg \mathrm{G} 2$ antibodies. Functional hFc gamma RIla polymorphism to IgG2. J Immunol 1993; 151: 1463-1472.

31. Bredius RG, Driedijk PC, Schouten MF, Weening RS, Out TA. Complement activation by polyclonal immunoglobulin G1 and G2 antibodies against Staphylococcus aureus, Haemophilus influenzae type b, and tetanus toxoid. Infect Immun 1992; 60: 4838-4847.

32. Breukels MA, Jol-van der Zijde E, van Tol MJ, Rijkers GT. Concentration and avidity of anti-Haemophilus influenzae type b (Hib) antibodies in serum samples obtained from patients for whom Hib vaccination failed. Clin Infect Dis 2002; 34: 191-197.

33. Hougs L, Juul L, Ditzel HJ, Heilmann C, Svejgaard A, Barington $\mathrm{T}$. The first dose of a Haemophilus influenzae type $\mathrm{b}$ conjugate vaccine reactivates memory $B$ cells: evidence for extensive clonal selection, intraclonal affinity maturation, and multiple isotype switches to IgA2. J Immunol 1999; 162: 224-237.

34. Abbas AK, Lichtman AH, Pillai S. Cellular and Molecular Immunology. In: Abbas AK, Lichtman AH, Pillai S (Editors), $B$ cell activation and antibody production. Philadelphia: Saunders Elsevier Press; 2007. p 232-234. 